\title{
Adaptive Agents for Sequential Vickrey Auctions with Homogeneous Goods
}

\author{
Huiye Ma \\ Center for Mathematics and Computer Science, Kruislaan 413, Amsterdam, The Netherlands
}

\begin{abstract}
In this paper, we aim to study the behaviour of the autonomous adaptive agents in sequential Vickrey auctions with homogeneous goods. We describe how to revise these adaptive agents, including ZI-C, ZIP, GD, and AA. The performance of these agents is evaluated by two sets of experiments. It turns out that truth-telling is not a dominant strategy; when it is easy for all the buyer agents to buy their goods, one should not tell his true value; when it is difficult to buy, one should not submit a random value; the performance of adaptive agents, such as ZIP and GD, is very good but cannot be guaranteed to be the best in various market environments. Through another set of experiments, the results demonstrate that $A A$ agents perform very well compared with the truth-telling agents and agents using other adaptive bidding strategies.
\end{abstract}

\section{Introduction}

Today, several auction mechanisms have been widely employed in electronic commerce, such as English auction, Vickrey auction, continuous double auction, etc. The Vickrey auction is well-known for the existence of the dominant strategy which is truth telling [8]. In our work, each Vickrey auction is assumed to sell one unit of the homogeneous goods. In order to relieve the burden of human traders in more and more complicated auctions, software agents are highly needed, which are representatives of human traders to achieve the humans' goals [11]. The dominant strategy (the best strategy to take, no matter what the others take) for an agent to adopt in such a single Vickrey auction is to bid his true value.

Auction designers have researched on the fundamental value of deadline in an auction mechanism and have applied it to many electronic commerce applications, such as eBay and Amazon [4] [5] [1]. In many real world situations it is essential to conclude a negotiation among agents within a fixed deadline. Aimed to resemble those practical market environments, in our Vickrey auction, there is a fixed deadline to regulate when the auction will finish. There- fore agents in such Vickrey auctions can count the time and submit their (sealed) bids when they want. After this single Vickrey auction is finished, another Vickrey auction will start because there may have more than one seller who wants to sell their goods in Vickrey auctions.

Many work has been done to design adaptive agents in continuous double auctions (CDAs), such as ZI-C [7], GD [6], ZIP [3], and AA [10]. Recently, Bagnall and Toft [2] have revised GD, ZIP, and ZI-C to be applied to the first price sealed bid (FPSB) auctions and second price sealed bid (SPSB or Vickrey) auctions. However, they have not described the revisions of AA agents nor compared the performance of AA agents with other agents. They have not analyzed the performance of various adaptive agents in dynamic market environments where supply and demand are fluctuating from time to time. Nevertheless, in real markets, the supply and the demand may change because people come and go. In addition, they have assumed each agent is aware of the number of competitive agents in the same market, which is usually unknown for markets in real life.

To the best of our knowledge, none of the other existing literature on Vickrey auctions gives a systematic treatment of adopting adaptive agents designed for continuous double auctions into sequential Vickrey auctions in dynamic market environments. However, it is possible to revise all these adaptive agents and enable them to learn through repeated competition in the sequential Vickrey auctions. More interesting questions are raised related to sequential Vickrey auctions. What is the performance of the truth-telling agents in the dynamic sequential Vickrey auctions? What is the performance of the revised adaptive agents when AA agents are included or not included in such market environments? Is it beneficial for agents to behave adaptively?

In order to answer the above questions, two sets of experiments are designed and carried out. In the first set of experiments, the performance of agents using the dominant strategies is compared with that of ZI-C, ZIP, and GD which are widely adopted in the literature. Therefore all these different agents are put together in the sequential Vickrey auction market. Later, AA strategy has been designed by Ma and Leung [10] for continuous double auctions and for sequential English auctions [9], which is formed based on 
eagerness and a set of heuristic rules with thresholds. Eagerness aims to simulate the feeling of a human trader in a sequence of repeated competition. In the sequential Vickrey auctions, eagerness can be naturally generated. Therefore, AA agents should be tested in the sequential Vickrey auctions as well. Both experimental results show that truthtelling is not a dominant strategy; when it is difficult to buy for all the buyers, ZI-C agents perform worse than agents using ZIP, GD, AA, and the truth-telling. In particular, experimental results of the second set of experiments show that AA agents performance very well compared with other adaptive agents and the agents using the truth-telling strategy.

The remainder of this paper is organized as follows. In section 2, the sequential Vickrey auctions with homogeneous goods are described and related work is given. Section 3 describes how to revise the adaptive agents for continuous double auctions into the sequential Vickrey auctions. In section 4 , the performance of ZI-C, ZIP, GD, and the dominant strategy is compared. AA strategy is then added into the experiments and experimental evaluation is given. Our conclusion and future work are presented in section 5.

\section{Preliminaries}

\subsection{Sequential Vickrey Auctions with Ho- mogeneous Goods}

A single Vickrey auction is a market where multiple buyers desire to buy one unit of goods and each buyer only has one chance to submit his bid in a sealed envelope to the auctioneer. The auctioneer starts the auction by announcing of the lowest acceptable price, denoted as $P_{\text {lowest }}$. All the rational buyers should submit their bids higher than or equal to the value of $P_{\text {lowest }}$. Otherwise, their bids will be ignored by the auctioneer.

For each unit of goods that a buyer desires to buy, there is a reservation price, above which a rational buyer will not buy the unit. The auction ends when all the buyers have submitted their bids. The buyer who placed the highest bid is the winner while paying the second highest bid submitted by others.

In a single Vickrey auction, only one unit of goods can be traded. If there are more sellers who want to sell their units of homogeneous goods, another Vickrey auction will begin after this Vickrey auction. The buyer which has traded successfully in the previous Vickrey auction will not join the later Vickrey auctions. All the rest buyers will continue to submit their bids in the later Vickrey auctions. A round is the trading period from the beginning of a single Vickrey auction to the end of this Vickrey auction. Within each round, at most one unit of goods is traded. When all the sellers have traded their units of homogeneous goods, a run is terminated. A run refers to the period for all the sellers to trade one after another.

\subsection{The Dominant Strategy in a Single Vickrey Auction}

In a single Vickrey auction, the dominant strategy is to bid his true reservation price to the auctioneer, denoted as $S_{\text {domi }}$.

\subsection{Bidding Strategies in CDAs}

The research into the design and analysis of bidding strategies for agents in CDAs started from early experimental economics work. Some representatives are selected to discuss below.

Gode and Sunder [7] proposed the first bidding strategies adopted by agents, ZI with Constraint (ZI-C) and ZI Unconstrained (ZI-U). A ZI-C buyer submits a bid which is a random value, larger than the lower range of the market and less than the reservation price of this unit of the good. ZI-C agents are widely adopted as benchmark agents because of their simplicity during implementation.

Cliff and Bruten [3] further designed "zero-intelligenceplus" (ZIP) agents by employing an elementary form of machine learning and a set of heuristic rules. Each ZIP agent has a profit margin which determines the difference between the agent's reservation price and the ask or bid submitted. The main idea is that if there was a transaction in the last round, the agent would increase his profit margin in the current round if his desired bid was higher than the transaction price. If there was no transaction, the agent would decrease his profit margin in the current round.

Gjerstad and Dickhaut [6] proposed a more sophisticated bidding strategy utilizing two belief functions, often referred to as GD. A GD agent records all the asks and bids in the history occurring in the last several rounds. From the history, an agent computes a subjective belief of a bid or ask being accepted. The agent can then calculate the expected utility of the bid or ask. The bid or ask corresponding to the highest expected utility is submitted to the market. By utilizing the subjective belief, the agent is sensitive to the fluctuation of the dynamic market all the time.

Recently, Ma and Leung [10] have proposed an adaptive attitude (AA) bidding strategy for agents in CDA markets. The AA strategy exploits both the short term and the long term attitudes of an agent, and utilizes a threshold based method with heuristic rules in bid determination. Eagerness should be affected by an agent's feeling in the past several rounds and in the last run as well. Eagerness acts as an indicator of the current supply and demand relationship from the agent's own point of view. 


\section{Agents for Sequential Vickrey Auctions with Homogeneous Goods}

When we look on a single Vickrey auctions as a double auction where many buyers submit their bids and only one seller (auctioneer) stays in the market without giving any asks, it is meaningful to introduce the agents from CDAs into the sequential Vickrey auctions. Certainly, we cannot simply consider the sequential Vickrey auction as a special case of CDAs, because the information available to the agent, and hence the learning problem, is fundamentally different. In double auctions, an agent receives a stream of data of asks, bids, and transactions. For sequential Vickrey auctions, each agent is only aware of the lowest acceptable price announced by the auctioneer, whether he is the winner or not, and the price the winner must pay. The sealed bidding nature prevents agents from observing competitors' bids. Hence all the agents in sealed-bid auctions are only able to infer and learn useful information once the auction has ended.

In this Section, we describe how we revise ZI-C, ZIP, $\mathrm{GD}$, and AA strategies for sequential Vickrey auctions from the perspective of buyer agents.

\subsection{ZI-C Buyer Agents}

For a ZI-C buyer agent $i$, he will generate his bid which is a random value from the range $\left[P_{\text {lowest }}, v_{i k}\right]$.

\subsection{ZIP Buyer Agents}

Since all the buyer agents in the sequential Vickrey auctions will not submit a bid lower than the lowest acceptable price $P_{\text {lowest }}$, it is reasonable to set a lower bound $P_{\text {lowest }}$ to the transaction price in the last round and the last bid in the last round.

In the Vickrey auction, each agent submits his bid to the auctioneer in a secret way and the winner is the one who submits the highest bid and pays the second highest bid as the transaction price, $P_{t}$. Therefore, the value transaction price $P_{t}$ is different from the actual value of the highest bid $P_{\text {highest }}$. Only the winner agent will know the exact value of $P_{\text {highest }}$. The rest agents only know that the value of $P_{t}$ and the relationship of $P_{\text {highest }}>P_{t}$. The unknown value of $P_{\text {highest }}$ will bring difficulty to the adjustment of the profit margin. For the winner agent, he will use the same way to adjust the profit margin as described by Cliff and Bruten [3]. For the rest agents, we divide them into three categories which are different from the revisions by Bagnall and Toft [2]. One is risk-averse who always try to avoid the risk of losing the next auction by increasing the target price, denoted as $Z I P_{r a}$. Another is risk-seeking who still want to lower the target price so as to pursue high profit in each auction, denoted as $Z I P_{r s}$. The third category is risk-neutral who will not change the target price and is denoted as $Z I P_{r n}$. Here we focus on the risk-averse ZIP buyer agents in the sequential Vickrey auctions. For the performance of risk-seeking and risk-neutral ZIP buyers, it is still an interesting issue in our future work.

\subsection{GD Buyer Agents}

GD agents for CDAs form a strategy based on the recent history of bids and asks. However, because it is not available for each agent to know the rejected bids in Vickrey auctions, the belief function becomes invalid in Vickrey auction. In order to solve this problem, the updated belief function has been proposed and demonstrated to be effective [2]. We follow their updated belief function to implement GD agents in our sequential Vickrey auctions.

\subsection{AA Buyer Agents}

The value of eagerness for AA agents is formed on the basis of the amount of transactions in several rounds or in consecutive runs. This is the same in the sequential Vickrey auctions. As to the heuristic rules to compute the bids, two thresholds $\alpha$ and $\omega$ are to be set.

In sequential Vickrey auctions, the threshold $\alpha$ has to be removed since there are no asks. In addition, the threshold $\omega$ has to be removed because all the bids from other agents in the current round are invisible to the agent. The available equation to compute bid in this situation is bid $=v_{i k}$ $\left(v_{i k}-P_{l l}\right) \times \mathrm{R} 3$. Here $v_{i k}$ is the reservation price, $P_{l l}$ is the lower range of acceptable prices in the market, and $R 3$ is a random real number.

In such sequential Vickrey auctions, repeated competition occurs for all agents. Hence eagerness can be kept for AA buyer agents because there is trading history for the AA agents to compute eagerness as in CDAs. In order to revise the AA strategy in a meaningful way and let it work properly, the random value of $R 3$ should be replaced by the meaningful value of eagerness; $P_{l l}$ should be replaced by introducing a variable, $P_{\text {thresh }}$. The value of $P_{\text {thresh }}$ is adjusted with different values of eagerness by employing three fuzzy logic rules, shown in Figure 1.

IF $F_{\text {eager }}$ is close_to 1.0 THEN $P_{\text {thresh }}$ is a small value; IF $F_{\text {eager }}$ is close_to 0.0 THEN $P_{\text {thresh }}$ is a large value; IF $F_{\text {eager }}$ is in-between THEN $P_{\text {thresh }}$ is a medium value;

Figure 1. The fuzzy rule base for adjusting $P_{\text {thresh }}$. 
The performance of ZI-C, ZIP, and GD has been extensively evaluated in the literature. Nevertheless, the issues of whether these adaptive agents can still work well in agent-based sequential Vickrey auctions, and which adaptive agent performs superiorly under different market situations, are interesting questions to be addressed. The experimental results below demonstrate the trend of the agent's profit with different strategies under various market environments.

\section{Experimental Evaluation of the Adaptive Buyer Agents in the Sequential Vickrey Auctions}

To evaluate the performance of agents employing the adaptive strategies and the dominant strategy, we design two sets of experiments respectively. In the first set of experiments, the profits gained by agents using strategy $X$ are compared. $X$ can be ZI-C, ZIP, GD, and the dominant strategy $S_{d o m i}$, where ZI-C, ZIP, and GD are the most widely cited strategies for agents based CDAs. In the second set of experiments, AA is added into the competition with ZI-C, ZIP, GD, and $S_{d o m i}$.

\subsection{Experimental Setup}

In each round, a buyer is endowed with one unit of the goods whose reservation price is independently and uniformly drawn from $[3.1,3.3]$. The value of the lowest acceptable price of the market, $P_{\text {lowest }}$, is randomly generated from the range $[1.1,1.3]$ and broadcast to all the agents. The thinking time that a buyer agent is allowed to elapse before submitting a bid is specified as a randomly distributed variable with a range $[1,2000]$. The fixed deadline to terminate a round is 20000 time units. In order to measure how well an agent performs in the sequential Vickrey auctions, we evaluate his performance by the profit he gains. For a buyer $i$, the total profit on all $t$ units bought in a run is $\sum_{k=1}^{t}\left(v_{i k}-P_{i k}\right)$ where $v_{i k}$ is the reservation price and $P_{i k}$ is the value of the transaction price. In the sequential Vickrey auctions, $P_{i k}$ is determined by the value of the second highest bid. An agent's profit is calculated as the sum of the total profits in 1000 runs.

In the first set of experiments, there are three different stages. In the first stage, there are totally 1000 runs where in each run, 10 rounds are implemented one after another. In each round, many buyer agents compete for one single unit of the good. Each buyer wants to buy one unit of the good in each round. For example, in the first round of the first run, 20 buyers compete for one unit of the good. Finally one buyer makes a transaction successfully. Then this buyer will leave the current run. In the second round of the run, the rest 19 buyers will continue to buy what they desire to buy in the following 9 rounds. After 10 rounds are finished, another run will begin and 20 buyers will reenter the market. Therefore, the competition during these 1000 runs in the first stage is very ferocious.

After these 1000 runs, we increase the amount of rounds per run to be 20 . This means that all the 20 buyer agents need to compete for 20 units of the goods in one run. There are altogether 1000 runs serially. It is obvious that in these 1000 runs, the competition is not as ferocious as the previous 1000 runs.

In the final stage, there are 40 rounds in each run. Hence, all the 20 buyers are provided 40 units of the good to buy while the buyers only desire to buy 20 units in all. Any buyer can take his time to buy the unit he desires. The competition is not ferocious at all.

In the second set of experiments, there are three stages as well which are similar to those in the first set of experiments. The difference is that there are 5 different kinds of buyer agents and each kind has 5 buyer agents. Consequently, the amount of rounds or demand in each run is 10 , 25 , and 50 every 1000 runs.

\subsection{Experimental Results in the First Set of Experiments}

Figure 2(a) shows the profit for ZI-C, ZIP, GD, and $S_{d o m i}$ buyer agents in the sequential Vickrey auctions. It can be observed that when the amount of rounds in each run is only $10, S_{d o m i}$ agents and ZIP agents perform the best when compared with ZI-C and GD agents.

If we take a look at Figure 2(b), it is shown that the amount of transactions achieved by $S_{d o m i}$ agents is the largest. The good profit of the $S_{d o m i}$ agents comes from this large amount of transactions and a good profit in each transaction. The large amount of transactions is determined by the strategy itself. Based on the strategy of the $S_{d o m i}$ agent, the agent will submit his bid, which is equal to his true reservation price. Therefore, the $S_{d o m i}$ agent will grab more opportunities to make transactions than other agents who tell lies if their reservation prices are the same. On the other hand, the transaction price is determined by the second highest bid submitted by another agent instead of the highest bid itself. This means that the profit for the winner is not determined by the winner himself, which is equal to various types of agents. Therefore the profit of $S_{d o m i}$ agents is one of the best in the first stage of the experiments since they have more opportunities to win and consequently gain a good profit in the end.

The performance of ZI-C agents is the worst in the first stage of the experiments. The amount of transactions of $\mathrm{ZI}-\mathrm{C}$ agents is the lowest as well. The lowest amount of transactions means that the bids submitted by $\mathrm{ZI}-\mathrm{C}$ agents 


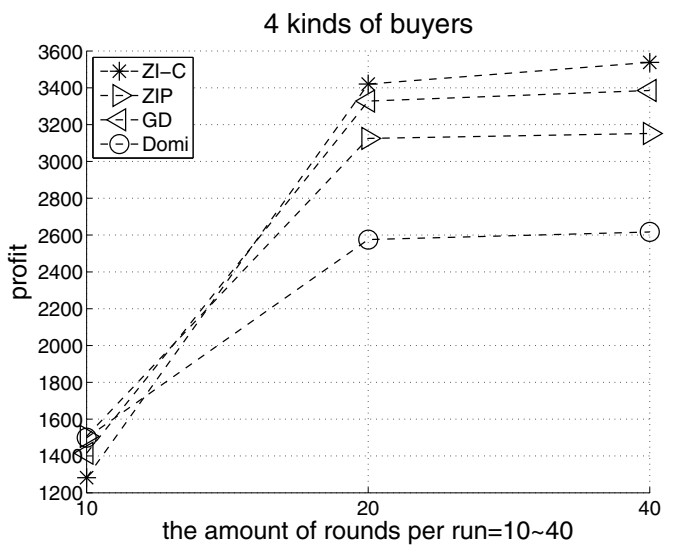

(a) the profit

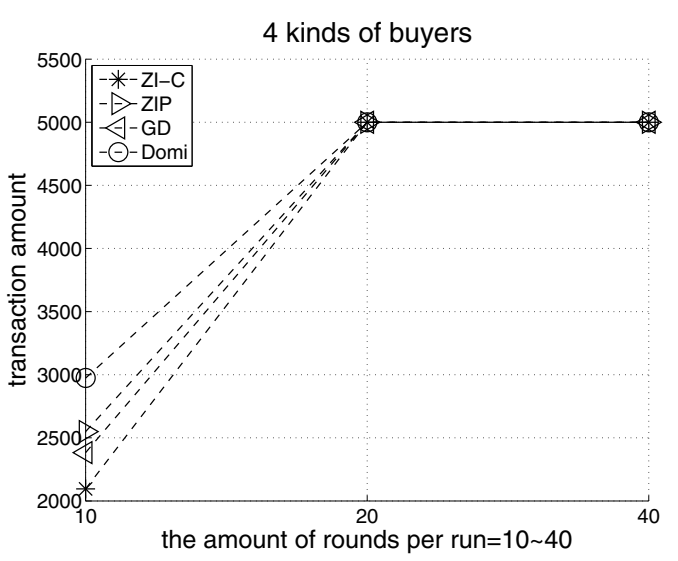

(b) the amount of transactions

Figure 2. Buyers adopting ZI-C, ZIP, GD, and $S_{d o m i}$ strategies compete with each other in the sequential Vickrey auctions. In every 1000 runs, there are 10,20 , or 40 rounds.

are not high enough to win a lot of transactions. In fact, in a very competitive market where the transaction price is determined by others, it has been illustrated by Figure 2 that the most important thing is to grab more transactions. As a result, the performance of ZI-C agents cannot be very good.

When the amount of rounds is 20 or 40 , the profit of $S_{d o m i}$ buyer agents is the worst. The reason is as follows. When the amount of rounds is 20 or 40 , every buyer agent has the opportunity to buy what they want. ZIP and GD agents have the ability to learn and behave adaptively to the market. The bids submitted by them are usually very low compared with those submitted by $S_{d o m i}$ agents. Hence ZIP, GD, or ZI-C agents will take enough profitable transactions and improve their profits. Moreover $S_{d o m i}$ agents are more liable to take those poor transactions because they always submit their true reservation prices. This will leave other profitable transaction opportunities to ZIP, GD, or ZI$\mathrm{C}$ agents. All these will eventually lead to the loss of the profit of $S_{d o m i}$ agents. In these two stages, compared with ZIP and GD agents, ZI-C agents achieve a very good performance. The reason is that the transaction price is decided by the second highest bid which is most likely submitted by ZIP or GD agents. Since ZIP and GD agents are adaptive and submit low bids in order to benefit themselves, these low bids will benefit ZI-C agents at the same time. Furthermore, ZI-C agents will not be disturbed by the bids of $S_{d o m i}$ agents while GD and ZIP agents do.

Throughout these three stages, the profit of ZIP and GD agents are neither the best nor the worst. The reason behind is that they can behave adaptively to the dynamic market by adjusting their bids from time to time. Nevertheless, in such Vickrey auctions, the transaction price is determined by the second highest bid. If these adaptive agents submit a high bid, they are more liable to grab more transaction opportunities but the profit in each transaction will be probably low unless the second highest bid is very small. If these agents submit a low bid aiming to improve the profit in each transaction, their opportunities to win will be low and if they lose their low bid will benefit other agents. In the end, this will lead to the profit of these adaptive agents to be not so high.

\subsection{Experimental Results in the Second Set of Experiments}

When we add AA buyer agents into the market, the result shown in Figure 3(a) is quite similar with that of Figure 2(a). If there are only 10 rounds per run, the profit of AA, ZIP, and $S_{d o m i}$ agents in Figure 3(a) are the best while ZI$\mathrm{C}$ agents perform the worst as in Figure 2(a). AA agents perform very well because the agents can know whether it is difficult for them to buy the goods by means of eagerness. Consequently they will adjust the value of $P_{\text {thresh }}$ according to the dynamic market environments. Therefore the effectiveness of eagerness is demonstrated by the good performance of AA agents. The reason for the good performance of $S_{d o m i}$ agents and the bad performance of ZI-C agents is similar to that in Section 4.2.

If there are 25 rounds or 50 rounds in each run, the profit of the AA agents is still very good compared with other agents. The outstanding performance of the AA agents again results from eagerness and $P_{\text {thresh }}$. In this situation, the value of eagerness is close to 1.0 which tells the agent that it is now easy for him to have transactions and he should be eager for more profit in each transaction. In addition, eagerness will guide the AA agent to adjust the value of $P_{\text {thresh }}$ to become smaller. Eventually the agent will generate a small bid which will leave a lot of profit to himself in each transaction. It is also true that such small bid 


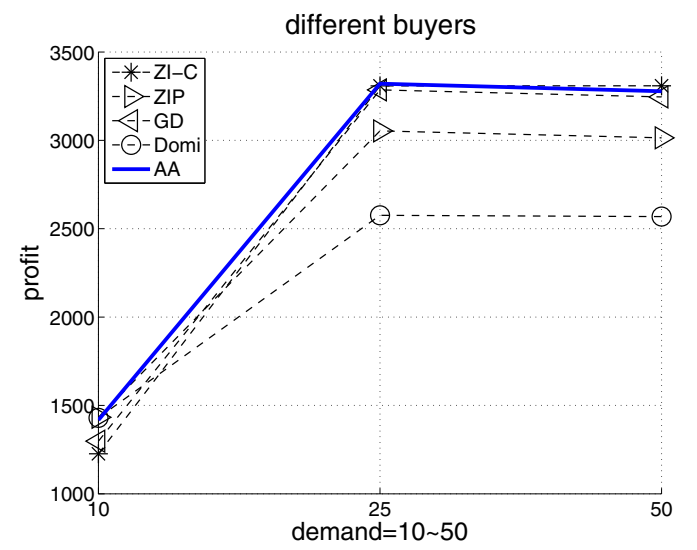

(a) the profit

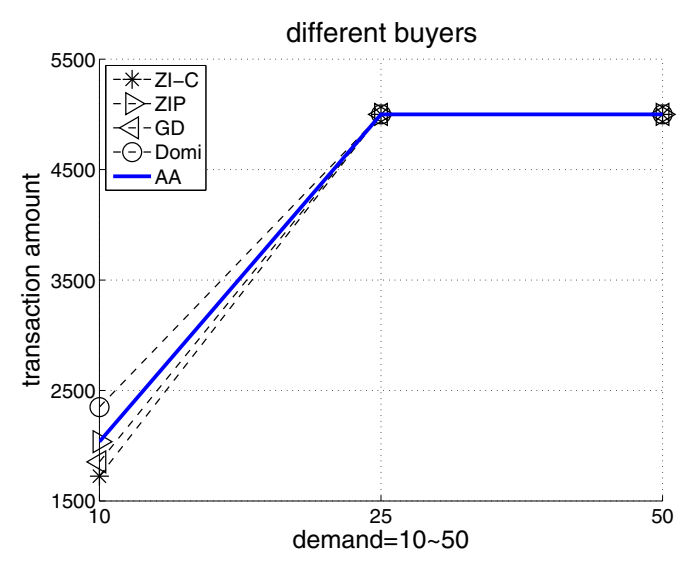

(b) the amount of transactions

Figure 3. Buyers adopting ZI-C, ZIP, GD, $S_{\text {domi }}$, AA strategies compete with each other in the sequential Vickrey auctions. In every 1000 runs, there are 10,25 , or 50 rounds.

may sometimes benefit other agents, such as ZI-C agents, which is shown in Figure 3(a) with demand $=50$. Finally, the AA agents and ZI-C agents will not be affected by the $S_{\text {dom } i}$ agents. However, ZIP agents and GD agents are still disturbed by the $S_{d o m i}$ agents especially when the $S_{d o m i}$ agents won the previous rounds of transactions.

\section{Conclusion and Future Work}

We present how to revise ZI-C, ZIP, GD, and AA strategies such that they can work properly in the sequential Vickrey auctions. Experimental results show that when it is difficult to have transactions, the performance of ZI-C agents is the worst; otherwise $S_{d o m i}$ agents perform worst. Sometimes ZI-C agents perform best if it is easy to trade because
ZIP and GD agents are affected by the $S_{d o m i}$ agents and lose profit. To test the performance of AA agents in the sequential Vickrey auctions, we put ZI-C, ZIP, GD, $S_{d o m i}$, and AA agents in the market and carry out another set of experiments. The performance of AA agents is demonstrated to be very well compared with other agents. There are more interesting questions to be addressed, i.e., how the ZIP or GD agents are disturbed by other agents in the market? in which scenarios the performance of ZIP, GD, or AA agents can be the best. We shall investigate more on these unaddressed questions in the future.

\section{Acknowledgments}

The work described in this paper was supported by the NWO project DIACoDeM, No 642.066.604.

\section{References}

[1] O. Axel and A. E. Roth. Late and multiple bidding in second-price internet auctions: Theory and evidence concerning different rules for ending an auction. Games and Economic Behavior, 55:297-320, 2006.

[2] A. Bagnall and I. Toft. Autonomous adaptive agents for single seller sealed bid auctions. Autonomous Agents and Multi-Agent Systems, 12(3):259-292, 2006.

[3] D. Cliff and J. Bruten. Minimal-intelligence agents for bargaining behaviors in market-based environments. Technical Report HP-97-91, Bristol, UK, Aug. 1997.

[4] E. David, R. Azoulay-Schwartz, and S. Kraus. Bidders' strategy for multi-attribute sequential english auction with a deadline. In Proceedings of the 2nd International Joint Conference on Autonomous Agents and Multiagent Systems, pages 457-464, New York, NY, USA, 2003. ACM.

[5] R. F. Easley and R. Tenorio. Jump bidding strategies in internet auctions. Management Science, 50(10):1407-1419, 2004.

[6] S. Gjerstad and J. Dickhaut. Price formation in double auctions. Games and Economic Behavior, 22:1-29, 1998.

[7] D. K. Gode and S. Sunder. Allocative efficiency of markets with zero-intelligence traders: Market as a partial substitute for individual rationality. Journal of Political Economy, 101(1):119-137, 1993.

[8] V. Krishna. Auction Theory. Academic Press, March 2002.

[9] H. Ma and H. fung Leung. Adaptive agents for sequential english auctions with a fixed deadline. In Proceedings of 2007 IEEE Joint Conference on E-Commerce Technology and Enterprise Computing, E-Commerce and E-Services, pages 29-38, Tokyo, Japan, July 2007.

[10] H. Ma and H. F. Leung. An adaptive attitude bidding strategy for agents in continuous double auctions. Electronic Commerce Research and Applications, 6(4):383-398, 2007.

[11] J. Rykowski and W. Cellary. Virtual web services: application of software agents to personalization of web services. In Proceedings of the 6th International Conference on Electronic Commerce, pages 409-418, New York, NY, USA, 2004. ACM. 